\title{
Simple Method for Determining Frictional Sliding Resistance and Frictional Energy Dissipation in Layered Ceramics
}

\author{
Desiderio Kovar, ${ }^{*, *}$ and Michael Thouless ${ }^{*, *}$ \\ Materials Science and Engineering Department, and Mechanical Engineering and Applied Mechanics Department, \\ University of Michigan, Ann Arbor, Michigan 48109
}

\begin{abstract}
A method is presented for measuring the frictional sliding resistance between cracked laminae in a layered ceramic. The first step in the test involves using a wedge to completely crack a weak interface along the length of the specimen. The cracked specimen is then loaded in three-point bending, and the load-deflection response is monitored. A deviation from linearity is observed when the load is sufficiently high for sliding to occur between the upper and lower halves of the specimen. The load at which this nonlinearity is first observed can be related to the interfacial sliding resistance. A model is then developed to relate the energy absorbed during a load-unload cycle to the frictional sliding resistance. The analysis is verified using a model system made from steel, and a ceramic composite made from silicon nitride and boron nitride.
\end{abstract}

\section{Introduction}

$\mathrm{M}$ ANY experimental techniques have been developed for measuring interfacial sliding resistance. These include fiber pushout ${ }^{1,2}$ and pullout $^{3}$ tests, and indirect techniques involving the measurement of hysteresis during tensile loading of matrix-cracked composites. ${ }^{4}$ All these tests are designed specifically for testing fiber-reinforced composites for which the mechanical properties are influenced by frictional sliding between the fiber and matrix. ${ }^{5}$ Although it is likely that frictional sliding resistance also influences the properties of layered ceramics, currently available methods for measuring this quantity are not amenable to the testing of simple, layered materials.

Because the mechanical properties of layered materials are generally far superior when tested in flexure than when tested in tension, ${ }^{6}$ it is likely that components made from layered materials would be primarily designed to experience flexural loading. In this paper, a simple test is developed that allows the frictional sliding resistance to be measured over a range of sliding displacements and in a geometry that is relevant to layered ceramics. This method also can be applied to the testing of laminated, fiber-reinforced composite materials when information about the sliding resistance between plies rather than between the fiber and matrix is desired. The feasibility of the method is demonstrated using a model system consisting of steel beams. The method is then applied to determine the sliding resistance between laminae of a layered ceramic made from silicon nitride separated by a weak layer of polycrystalline boron nitride.

\footnotetext{
A. G. Evans - contributing editor
}

Manuscript No. 191788. Received May 28, 1996; approved September 16, 1996. Supported by DARPA; administered by the Office of Naval Research under Contract No. N0014-95-0302.

"Member, American Ceramic Society.

${ }^{\dagger}$ Materials Science and Engineering Department.

${ }^{*}$ Mechanical Engineering and Applied Mechanics Department.

\section{Analysis}

\section{(1) Sliding Resistance}

The analysis of the sliding resistance and energy absorption during bending makes use of simple, Eulerian elastic beambending theory. All of the usual kinematic assumptions are made, ${ }^{7}$ and, in addition, it is assumed that the frictional sliding resistance for a given interface can be characterized by a single parameter, $\tau_{\mathrm{s}}$. It also is assumed that the interfaces have a finite fracture resistance and that interfacial cracking and interfacial sliding can be considered to be two separate phenomena. Although interfacial fracture resistance also can be measured using this specimen, ${ }^{8}$ only interfacial sliding is analyzed in this study.

A schematic illustration of the specimen used to measure sliding resistance is shown in Fig. 1. A crack is created by inserting a wedge into the side of a notched beam of height $H$ until it splits into two beamlets along a weak plane. The resulting beamlets have heights $h_{1}$ and $h_{2}$. Consider these two beamlets, of length $L$, width $b$, and heights $h_{1}$ and $h_{2}$, reassembled one on top of the other. If the specimen is bent in threepoint flexure by a force $P$, a shear stress distribution develops through the thickness of the beam. If no relative sliding occurs between the beamlets, the shear stress on the interface and within the span has a magnitude given by ${ }^{7}$

$$
\tau=\frac{3 P h_{1} h_{2}}{b\left(h_{1}+h_{2}\right)^{3}}
$$

Provided that the sliding resistance is greater than this shear stress, the interface sticks and the beamlets behave as if they were a monolithic beam of height $H=h_{1}+h_{2}$. The deflection $y$ at the center of the beam is then given by

$$
y=\frac{P s^{3}}{48 E I_{\mathrm{k}}}
$$

where $s$ is the span between the lower supports, $E$ Young's modulus, ${ }^{\S}$ and $I_{\mathrm{k}}$ given by

\$n this case, both of the beamlets are made from the same material and, therefore, have the same Young's modulus, which is assumed to be isotropic. An appropriate correction can be made to this analysis if the beamlets have different elastic properties.

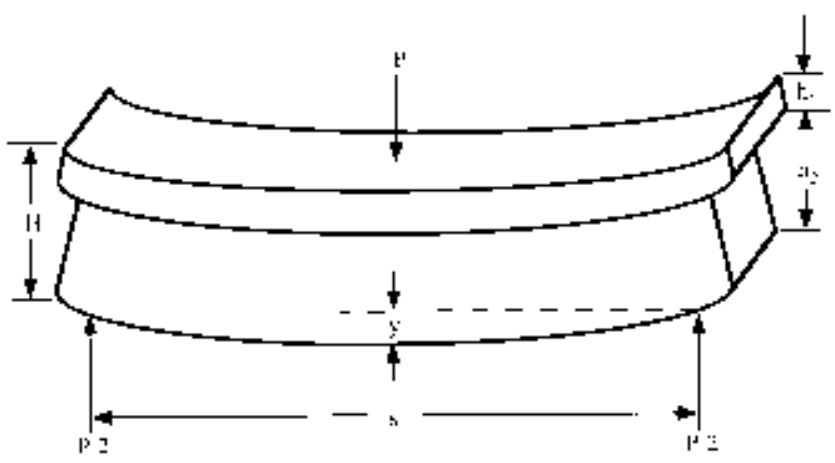

Fig. 1. Specimen used to measure interfacial sliding resistance in flexure consisting of two beamlets of height $h_{1}$ and $h_{2}$ and width $b$. 


$$
I_{\mathrm{k}}=\frac{b\left(h_{1}+h_{2}\right)^{3}}{12}
$$

If the shear stress on the interface exceeds the resistance to slipping, a change in the compliance of the beam occurs as the beams begin to slip. For a beam free of friction $\left(\tau_{\mathrm{s}}=0\right)$, the deflection of the cracked beam is again given by Eq. (2), where $I_{\mathrm{k}}$ is replaced by the effective second moment of inertia during slipping, $I_{\mathrm{p}} \cdot{ }^{\mathrm{IT}}$

$$
I_{\mathrm{p}}=\frac{b\left(h_{1}^{3}+h_{2}^{3}\right)}{12}
$$

For the purposes of this analysis, it is assumed that, once sliding has begun, the magnitude of $\tau_{\mathrm{s}}$ does not change the effective compliance of the cracked beam. In this case, $I_{\mathrm{p}}$ can be used as the effective second moment of inertia, independent of the magnitude of $\tau_{s}$. In addition, it is assumed that sliding commences when the shear stress acting on the interface between the supports exceeds the resistance to slipping. The load at which slipping begins, $P_{1}$, is determined by equating Eq. (1) to the sliding resistance. The sliding resistance therefore can be calculated directly from the specimen geometry and the load at which a deviation from linearity is observed in the loaddeflection response; it is given by

$$
\tau_{\mathrm{s}}=\frac{3 P_{1} h_{1} h_{2}}{b\left(h_{1}+h_{2}\right)^{3}}
$$

\section{(2) Energy Absorption}

Energy absorption due to frictional sliding can be measured directly from the hysteresis that occurs during a load-unload cycle. However, to design ceramics capable of high energy absorption, an ability to predict energy adsorption from interfacial properties is necessary. In this section, a method is developed to predict energy absorption from the frictional sliding resistance for a beam containing a single, interfacial crack.

Consider the load-deflection response during a load-unload cycle for the specimen shown in Fig. 1. When loading commences, no slipping occurs between the beamlets, and the loaddeflection response is linear. When the load reaches $P_{1}$, the interfacial shear stress reaches $\tau_{\mathrm{s}}$, and sliding begins at a constant shear stress. At this point, a deviation in the linear loaddeflection response occurs. Loading is continued until an arbitrary load $P_{\mathrm{m}}$ is reached, at which point unloading is begun. Upon unloading, the interfacial shear stress decays and becomes negative. Because the magnitude of the shear stress is initially less than resistance to sliding, the interface sticks until $\tau=-\tau_{\mathrm{s}}$, at which point reverse slip begins. The load at which reverse slip commences is designated $P_{\mathrm{u}}$. A schematic of the load-deflection curve is shown in Fig. 2 for a single loadunload cycle.

The energy dissipated during the complete cycle is the area within the hysteresis loop. This area is given by

$$
\begin{aligned}
W= & \int_{0}^{y_{1}}\left(m_{\mathrm{k}} y\right) \mathrm{d} y+\int_{y_{1}}^{y_{\mathrm{m}}}\left(m_{\mathrm{p}} y+b_{2}\right) \mathrm{d} y-\int_{y_{\mathrm{u}}}^{y_{\mathrm{m}}}\left(m_{\mathrm{k}} y+b_{3}\right) \mathrm{d} y \\
& -\int_{0}^{y_{\mathrm{u}}}\left(m_{\mathrm{p}} y\right) \mathrm{d} y
\end{aligned}
$$

where $m_{\mathrm{p}}$ and $m_{\mathrm{k}}$ represent the slopes of the load-deflection curve in the slipping and sticking regimes, respectively; $b_{2}$ and $b_{3}$ are the ordinate-intercepts for the slipping regime on loading and the sticking regime on unloading, and are given by

\footnotetext{
${ }^{\mathbb{I}} I_{\mathrm{p}}$ is calculated by equating the deflection of the upper and lower beamlets at the center of the beam.
}

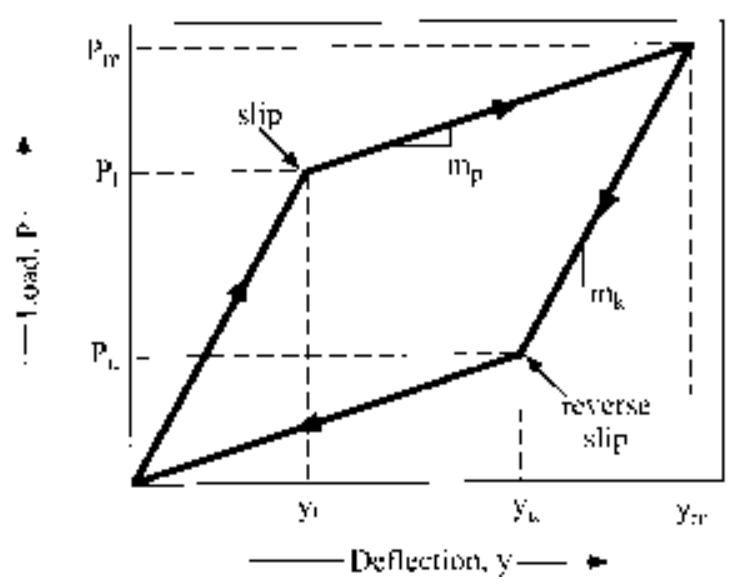

Fig. 2. Predicted load-deflection response for a cracked specimen loaded in flexure. Slipping between the beamlets occurs on loading at a load $P_{1}$ and on unloading at a load $P_{\mathrm{u}}$. Energy absorbed during the loadunload cycle is given by the area within the loop.

$$
\begin{aligned}
& m_{\mathrm{k}}=\frac{4 E b}{\Sigma^{3}} \\
& m_{\mathrm{p}}=\frac{4 E b\left(1-3 \eta+3 \eta^{2}\right)}{\Sigma^{3}} \\
& y_{1}=\frac{T \Sigma^{3} H}{12 \eta(1-\eta)} \\
& y_{\mathrm{u}}=\frac{P_{\mathrm{m}}-P_{1}}{m_{\mathrm{p}}} \\
& y_{\mathrm{m}}=y_{\mathrm{u}}+y_{1} \\
& b_{2}=P_{\mathrm{m}}-m_{\mathrm{p}} y_{\mathrm{m}} \\
& b_{3}=P_{\mathrm{m}}-m_{\mathrm{k}} y_{\mathrm{m}}
\end{aligned}
$$

where $\eta=h_{1} / H, T=\tau_{\mathrm{s}} / E$, and $\Sigma=s / H$.

Simplifying Eq. (6), it can be shown that the energy absorbed because of frictional sliding during a load-unload cycle is given by

$$
W=y_{\mathrm{u}} y_{1}\left(m_{\mathrm{p}}-m_{\mathrm{k}}\right)+b_{2} y_{\mathrm{u}}-b_{3} y_{1}
$$

In terms of the normalized parameters, $\eta, T$, and $\Sigma, W$ is given by

$$
W=\frac{E b H^{2} T \Sigma^{3}[T+3 \Pi \eta(\eta-1)]}{12\left[\eta(\eta-1)\left(1-3 \eta+3 \eta^{2}\right)\right]}
$$

where $\Pi$ is the normalized peak load, $P_{\mathrm{m}} / E b H$. Equation (9) shows that the amount of energy that is dissipated during a load-unload cycle is dependent on the span-to-depth ratio of the beam, maximum load through which the specimen is cycled, height ratio of the beamlets, and frictional sliding resistance. In Fig. 3(a), the normalized hysteresis energy is plotted as a function of the normalized sliding resistance for different values of the normalized peak load attained during the loadunload cycle. For a given value of $P_{\mathrm{m}}$, the energy dissipated by frictional sliding depends on $\tau_{\mathrm{s}}$. When the sliding resistance is very small, little energy is absorbed. Similarly, when the sliding resistance is very large, the sliding displacements are small, and, again, little energy is absorbed. The value of normalized sliding resistance that maximizes $W$ for a given value of the normalized peak load, $P$, is given by

$$
\frac{\tau_{\mathrm{s}}}{E}=\frac{3 \Pi \eta(1-\eta)}{2}
$$

In practice, once delamination has occurred in a laminated composite, there is an upper bound on the maximum load that can be subsequently supported. This peak load is determined 


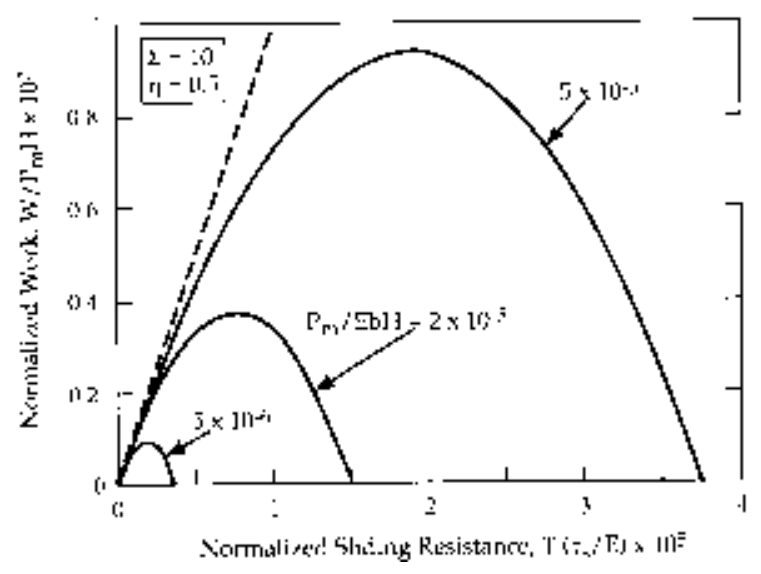

(a)

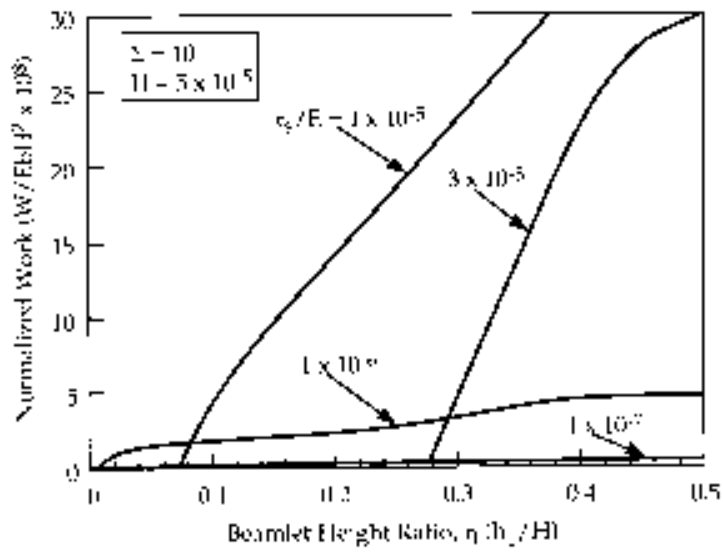

(b)

Fig. 3. (a) Predicted, normalized hysteresis energy plotted as function of the normalized maximum load attained during a load-unload cycle ((---) predicted response as $P_{\mathrm{m}}$ approaches infinity). (b) Predicted, normalized hysteresis energy plotted versus the beamlet height ratio for different values of the normalized sliding resistance.

by the yield or fracture strength of the individual layers. Figure 3(a) shows that designing a composite to maximize energy absorption involves tailoring the sliding resistance to match $P_{\mathrm{m}}$, as indicated by Eq. (10). For example, if the strength of the layers is increased, higher values of the sliding resistance are required to maximize energy dissipation.

In Fig. 3(b), the normalized hysteresis energy is plotted as a function of the beamlet height ratio, $\eta$, for different values of the normalized sliding resistance, $\tau_{\mathrm{s}} / E$. Figure $3(\mathrm{~b})$ shows that, when the height ratio is very small, insufficient shear stresses are developed on the interface for sliding to occur. Above this critical value of the height ratio, energy dissipation increases rapidly with the height ratio. However, the critical value of the height ratio at which sliding (and, therefore, energy absorption) begins, increases with the sliding resistance. Thus, specimen geometry is important in maximizing energy absorption in layered composites. If the height ratio is too small, or the sliding resistance is too large, little energy is absorbed.

\section{Experimental Procedure}

For the initial tests, a model system consisting of three sets of beams prepared from A2 tool steel was investigated. All the beams were of length $L=52.2 \mathrm{~mm}$ and width $b=3.7 \mathrm{~mm}$. One specimen was a monolithic bar of height $H=5.0 \mathrm{~mm}$. A second set of specimens consisted of two beamlets with $h_{1}=$ $h_{2}$, and a third set of bars had $h_{1}=4 h_{2}$; the total height for both sets was fixed at $H=h_{1}+h_{2}=5.0 \mathrm{~mm}$. One surface on each beamlet was polished, whereas the opposite surface was ground using a 220 grit grinding wheel. The sliding resistance between the beamlets was varied by placing either the polished surfaces or the ground surfaces in contact with one another. Additional experiments were conducted using a thin layer of boron nitride powder (HCP Grade, Advanced Ceramics Corp., Lakewood, $\mathrm{OH})$ placed on the interface to act as a solid lubricant.

Another set of experiments was performed in which the sliding behavior of a ceramic sandwich specimen made from silicon nitride and boron nitride was investigated. The specimen consisted of a thin layer of interphase material (75 vol\% hexagonal boron nitride and 25 vol\% silicon nitride) with a thickness of $\sim 250 \mu \mathrm{m}$ sandwiched between thick layers of silicon nitride. Details regarding the fabrication of this specimen are given elsewhere. ${ }^{9}$ The specimen was cracked by inserting a wedge into a notch that was cut into the specimen and extended into the interphase (Fig. 4). This drove a crack completely through the interphase, creating two beamlets with heights of 1.50 and $1.68 \mathrm{~mm}$. Because the crack extended in the interphase rather than at one of the silicon nitride-boron nitride interphases, the sliding surfaces of both beamlets consisted of polycrystalline, hexagonal boron nitride.

All the specimens were tested in a fully articulating, threepoint bend fixture with free-rolling, hardened, steel pins (Model W2662-2, Instron Corp., Canton, MA) using a screw-driven testing machine (Model 4483, Instron Corp.) and a load cell with a capacity of $5000 \mathrm{~N}$. The span between the lower load points was $40 \mathrm{~mm}$. The machine was run in displacement control at a crosshead displacement rate of $0.1 \mathrm{~mm} / \mathrm{min}$ during both loading and unloading. Specimen deflection was monitored using a linear-variable differential transformer (LVDT) deflectometer (Model S2-8321, Instron Corp.) with a resolution of $\pm 0.1 \mu \mathrm{m}$ located directly beneath the center loading point. Load and deflectometer-displacement data were collected at a rate of 5 points/s using a computerized data-acquisition system.

Because of settling that inevitably occurred between the specimen and load train during the initial stages of loading, it was more convenient to measure the sliding resistance from hysteresis loops during partial, rather than full, unloading cycles. This necessitated a modification to Eq. (6) to calculate the sliding resistance. To determine the sliding resistance during partial unloading, $\Delta P=P_{1}-P_{\min }$ was substituted into Eq. (5) for $P_{1}$, where $P_{\min }$ is the minimum load in the hysteresis loop. Similarly, on unloading, the sliding resistance was determined by substituting $\Delta P=P_{\mathrm{m}}-P_{\mathrm{u}}$ for $P_{1}$.

\section{Results}

\section{(1) Sliding Resistance-Steel Beams}

The load, applied over several cycles to two steel specimens with different height ratios, is plotted against the deflectometerdisplacement in Figs. 5(a) and (b). As a point of comparison,

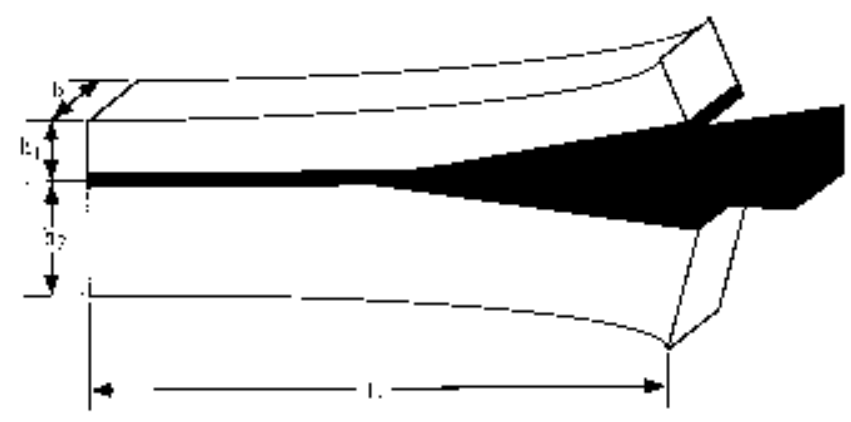

Fig. 4. Schematic illustration of the procedure used to crack a weak interface along the length of a beam using a wedge. 
load-deflection plots for a monolithic steel beam with the same total height as the beamlets also are shown. These plots show that the monolithic beam displays virtually imperceptible hysteresis. This indicates that there is minimal friction in the loading system at the contacts between the loading roller pins and the specimen surface. In contrast, all of the other specimens display measurable hysteresis loops, and the general shape of these loops is consistent with the theoretical shape shown in Fig. 2. Some settling of the specimen-load train is apparent in the curves at low loads; this is probably responsible for the slight residual load that is apparent upon complete unloading. For a given height ratio of the beamlets, the size of the hysteresis loops is dependent on the surface finish of the beams: The beams with boron nitride powder on the surface exhibit the narrowest loops, followed by the beamlets with polished surfaces, and then the beams with ground surfaces.

The sliding resistance, determined from the load-deflection curves, as described in Section III, is dependent on the condition of the sliding surface. The sliding resistance is $0.29-0.40$ MPa for the surfaces sprinkled with boron nitride powder, 1.01.5 $\mathrm{MPa}$ for the polished surfaces, and 1.1-1.7 $\mathrm{MPa}$ for the ground surfaces. For a given surface condition, similar values of the sliding resistance were measured when the height ratio of the beams was varied. For example, the sliding resistance for the beamlets sprinkled with powdered boron nitride with a height ratio of 1 is $0.29 \pm 0.04 \mathrm{MPa}$, whereas the sliding resistance for the beamlets with a height ratio of 0.2 is $0.40 \pm$ $0.10 \mathrm{MPa}$.

\section{(2) Energy Absorption during Bending-Steel Beams}

The energy absorbed during a single load-unload cycle was calculated using the model and compared to the measured value of the energy absorbed based on the area within a hysteresis loop. Because no fitting parameters were used, this provided an independent method of verifying the accuracy of the model with experimental data.

Single hysteresis loops for the beamlets with height ratios of 1 and 0.2 and with ground surfaces contacting are shown in Figs. 6(a) and (b). Also plotted on these two figures are the theoretical predictions based on the model developed in Section II(2). The theoretical specimen deflection at $P_{1}, P_{\mathrm{m}}$, and $P_{\mathrm{u}}$ have been calculated from the sliding resistance, and the slopes of the lines have been calculated directly from the specimen geometry using Eqs. (7a)-(7e) with an appropriate correction being made for machine compliance (see Appendix). Comparing the data to the theoretical predictions shows that the general trends apparent in the data are matched by the prediction. For example, the loading and unloading slopes appear to be similar, and a clear change in compliance occurs on loading and unloading. The transition between the slopes, however, is less sharp than predicted by the theory. The reasons for this are discussed in Section V. Despite these minor differences between the model and the prediction, most of the essential features apparent in the data appear to be captured by the model.

For the steel beamlets with ground sliding surfaces and a height ratio of 1 (Fig. 6(a)), the measured value of energy absorption is $1.32 \mathrm{~mJ}$ during a single load-unload cycle compared to the predicted value of $1.43 \mathrm{~mJ}$. For the ground steel beamlets with a height ratio of 0.2 (Fig. 6(b)), the measured value of the hysteresis energy is $0.63 \mathrm{~mJ}$ compared to the predicted value of $0.72 \mathrm{~mJ}$. The measured and predicted values of the hysteresis energy are shown in Fig. 7 as a function of the load change (the difference between the maximum load and the minimum load attained during the loading cycle) for the ground-steel beamlets. Agreement between the predicted and measured values of the hysteresis energy is generally good, and the trends observed in the data also are apparent in the predictions. As predicted from the theory, increasing the sliding resistance, increasing the magnitude of the load drop, or decreasing the beamlet height ratio all result in an increase in the hysteresis energy.

\section{(3) Sliding Resistance-Silicon Nitride-Boron Nitride}

A load-deflection plot for a silicon nitride-boron nitride specimen that was cyclically loaded is shown in Figs. 8(a) and (b). As with the steel specimens, the general shape of the curves is consistent with theoretical predictions. The measured sliding resistance for this bar has been determined to be $0.65 \pm$ $0.09 \mathrm{MPa}$. This is smaller than that measured for the polished steel bars, but larger than that for the steel bars with boron nitride powder sprinkled on the sliding surfaces.

A scanning electron microscopy (SEM) micrograph of a sliding surface created by fracturing a layered ceramic bar is shown in Fig. 9. Because fracture occurred in the boron nitridecontaining interphase rather than at the interface, both sliding surfaces consisted primarily of boron nitride. Fracture occurred by cleavage of the hexagonal boron nitride grains along their weak basal planes. The platelet-shaped grains were aligned roughly parallel to the interface, and the fracture path followed the structure of the boron nitride flakes and resulted in a very rough fracture surface. During sliding, it is likely that this roughness provided mechanical interlocking until the platelets were dislodged or deformed, allowing the rough surfaces to slide over one another. The roughness of the sliding surfaces may have been responsible for the larger sliding resistance measured in this specimen compared to that measured for the

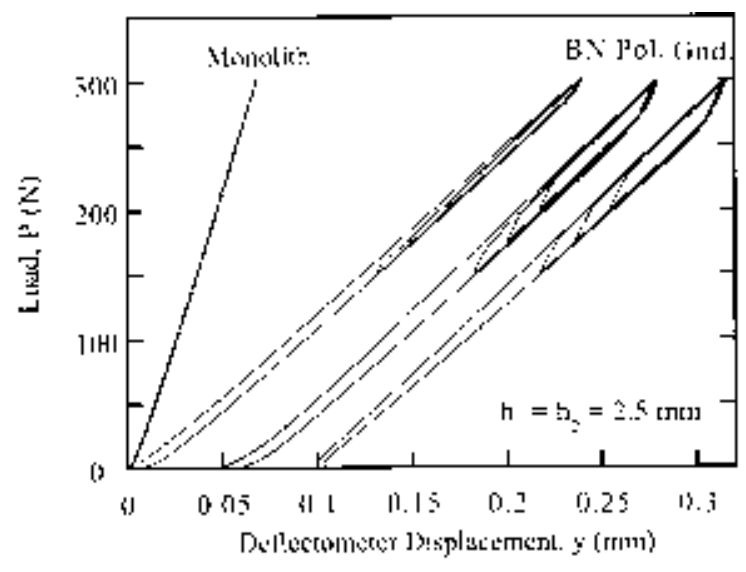

(a)

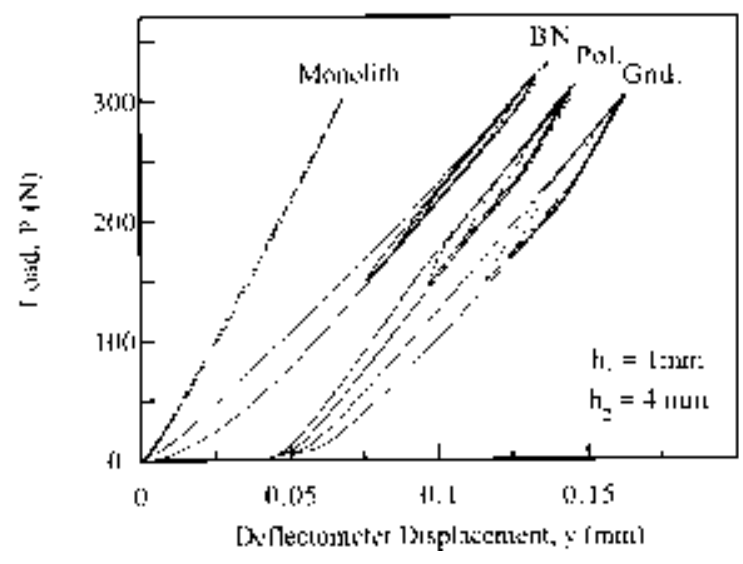

(b)

Fig. 5. Load-deflection response for steel beamlets with a height ratio $h_{2} / h_{1}$ of (a) 1 and (b) 4 that were repeatedly loaded and unloaded. Loaddeflection response for a solid beam of height $H=h_{2}+h_{1}$ also is shown in each plot. No hysteresis is observed in the solid beam but is appreciable in the beamlets. Width of the loops increases as the sliding surface is varied from powdered boron nitride (BN) to a smooth polished surface (Pol.), to a rough ground surface (Gnd.). 


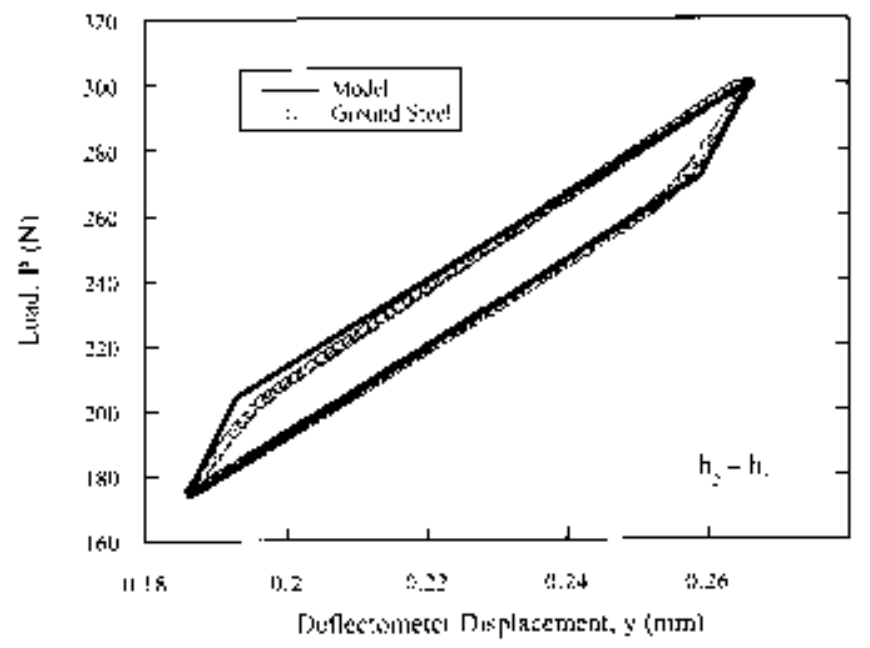

(a)

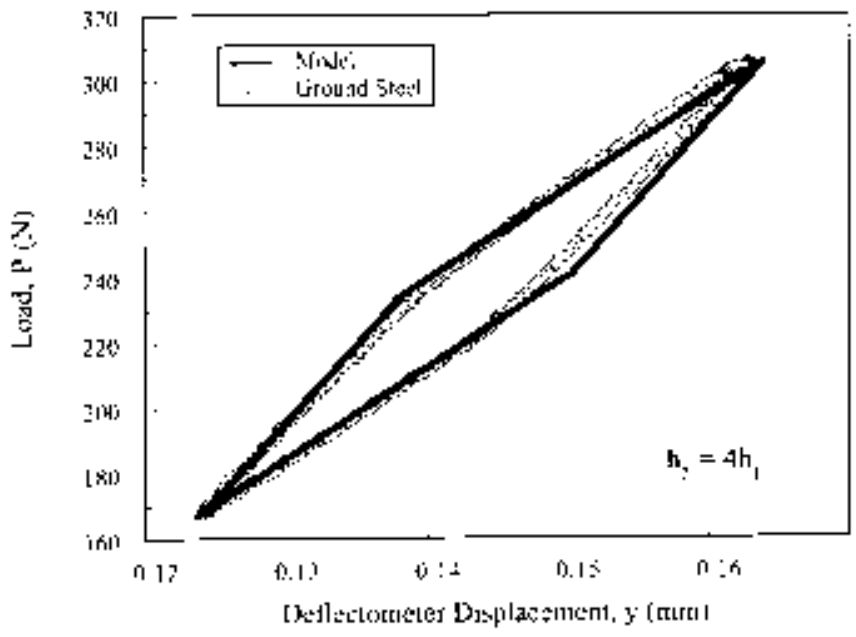

(b)

Fig. 6. Single hysteresis loops are shown for ground steel bars with a height ratio of (a) 1 and (b) 0.2 . Experimental data are points, and the predicted behavior based on the model is a solid line.

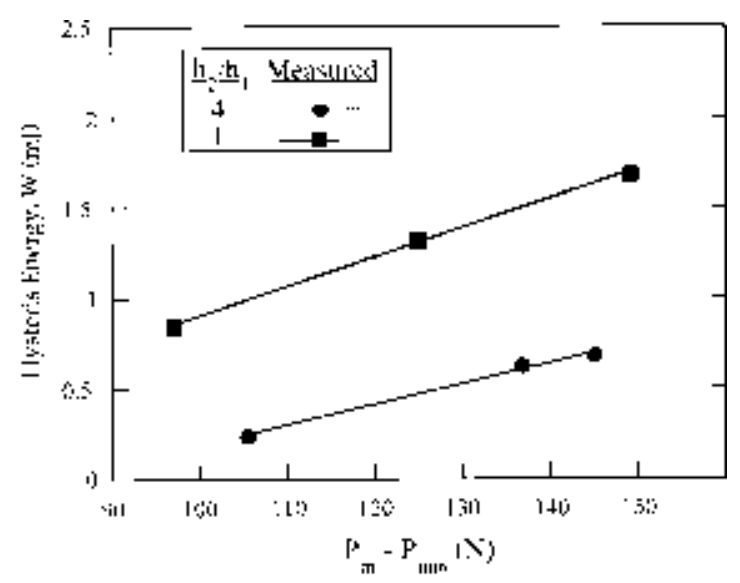

Fig. 7. Measured and predicted hysteresis energy for ground steel specimens with $\eta=1$ and $\eta=0.2$. Measured data are points, and shaded regions indicate the predicted hysteresis energy based on the sliding resistance using the model.

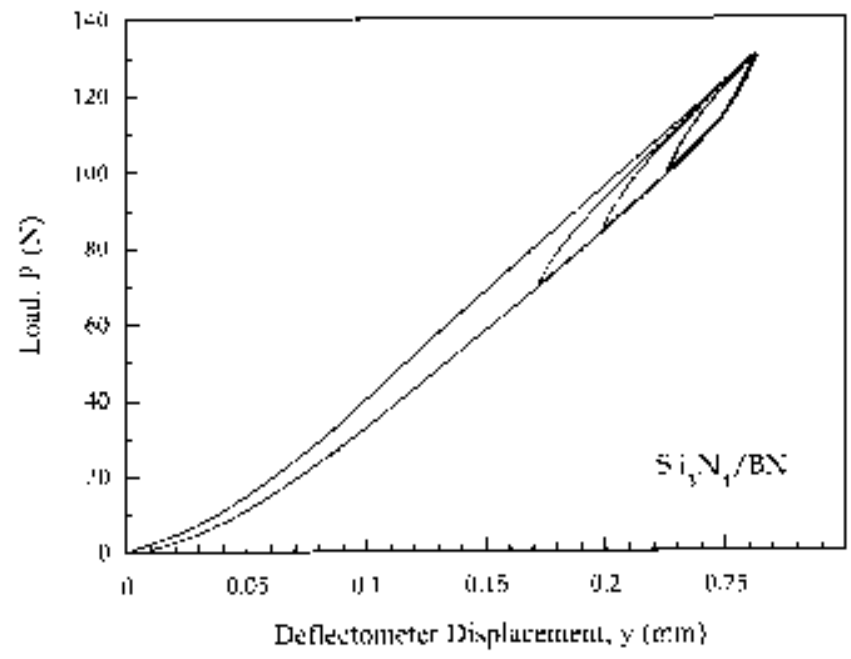

(a) steel bars sprinkled with loose boron nitride powder. This was consistent with previous experimental and theoretical studies that have shown that increased roughness of the fracture surface can increase the sliding resistance substantially. ${ }^{10,11}$

\section{(4) Energy Absorption during Bending-Silicon Nitride-} Boron Nitride

The measured value of the hysteresis energy for the cycle shown in Fig. 8(b) was $0.57 \mathrm{~mJ}$ compared to a predicted value of $0.66 \mathrm{~mJ}$ calculated from the interfacial sliding resistance. Similar agreement between the measured and predicted values of the sliding resistance was found for all the measured loops.

\section{Discussion}

\section{(1) Comparison of Results with Previous Results}

Because existing methods to determine frictional sliding resistance are designed for fiber-matrix systems rather than laminates, direct comparisons between the results of the current method and existing methods are difficult. The data that do exist for fiber-reinforced composites with boron nitride interfaces result in somewhat larger values of sliding resistance than

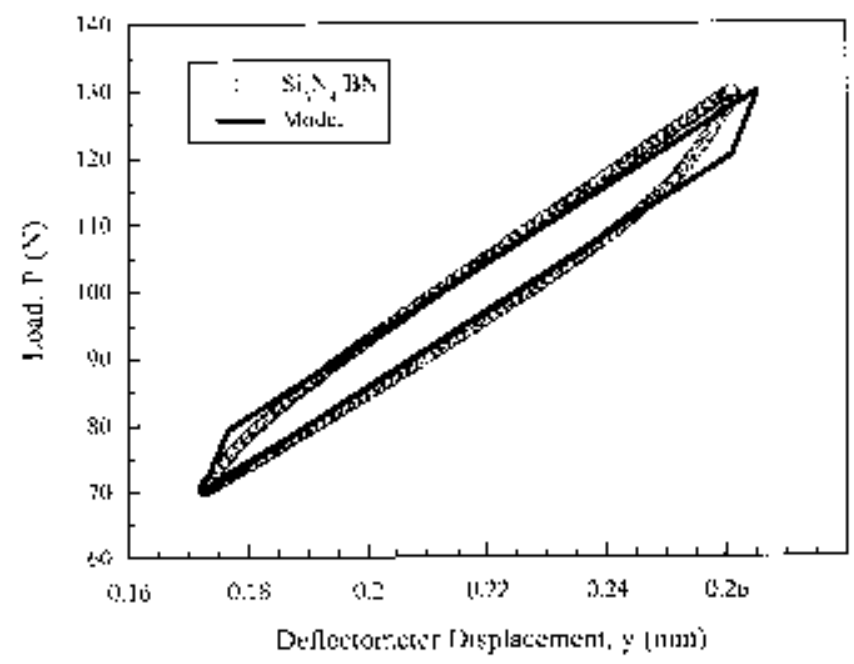

(b)

Fig. 8. Load-deflection response for silicon nitride-boron nitride specimen loaded and unloaded (a) several times and (b) for a single loadunload cycle. 


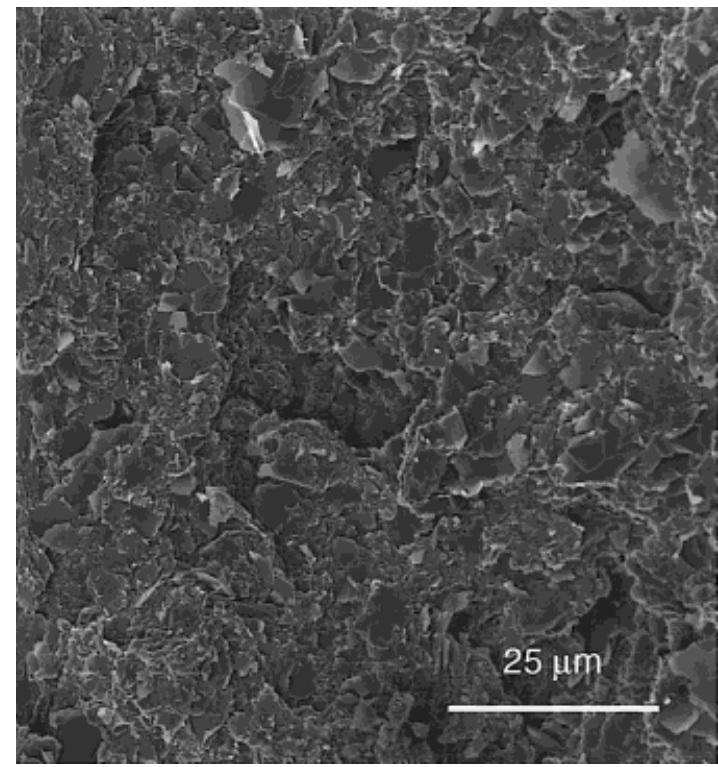

Fig. 9. SEM micrograph of sliding surface of silicon nitride-boron nitride specimen prior to testing, showing roughness of sliding surface caused by cleavage of boron nitride-platelet-shaped grains.

those found here. For example, Kumaria et al. ${ }^{12}$ have reported a value of $6.6 \mathrm{MPa}$ for the sliding resistance between a boron nitride-coated silicon carbide fiber in a zircon matrix measured using the fiber-pushout technique. However, unlike the case for layered materials, it is possible that residual stresses present because of thermal anisotropy between the fiber and matrix significantly influence the sliding resistance. For example, Kumaria et al. have reported axial, compressive residual stresses acting on the fiber-matrix interface of 3-4 GPa. Given that such residual stresses are not present at the sliding interface of a cracked layered ceramic and that normal loads acting on the interface because of the applied force at the loading pins are relatively modest $(\sim 2.5 \mathrm{MPa})$, the current values of sliding resistance appear reasonable.

\section{(2) Influence of Specimen Geometry on the Measurement of Sliding Resistance and Energy Absorption}

The geometry of the specimen can have a significant influence on errors in measuring the sliding resistance and the energy dissipation. Among the influences that are discussed in this section are the span-to-depth ratio, height ratio of the beamlets, and span-to-length ratio of the specimen.

The span-to-depth ratio influences the ratio of the normal stresses generated in the beamlets to the shear stress developed at the cracked interface. If the span-to-depth ratio is too large, the normal stresses that develop in the beam because of the bending moment crack or plastically deform the beamlets before slipping at the interface occurs. If the span-to-depth ratio is too small, slipping may occur at loads that are too low to detect. Thus, the optimum span-to-depth ratio must be determined for a given material and testing system.

The height ratio of the beamlets also influences the ratio of the shear stress to normal stress in a similar manner because the shear stress prior to the onset of slipping drops parabolicly away from the center of the beam. If the height ratio, $\eta$, is too small, cracking of the thinner layer may occur prior to slipping on the interface. The height ratio also influences the relative slope change between the sticking and slipping regimes on a load-deflection plot; the largest change in slope occurs when the height ratio is 1 . Although the height ratio should not influence the measured value of the sliding resistance, in practice it has been found that more consistent values of the sliding resistance are acquired when the change in slope is distinct.

It has been noted that the transition between the sticking and slipping regimes is somewhat more gradual than the sharp transition predicted by the model. This transition region can lead to significant errors in the measurement of energy dissipation because of frictional sliding. One possible explanation for the transition region involves the ratio of the length of the beams, $L$, to the span, $s$. Steif and Trojnacki ${ }^{13}$ have analyzed the predicted load-deflection response for a layered specimen containing a number of interfaces assumed to be weak in shear. They have found that this length-to-span ratio can have a significant influence on the load-deflection response. This is because, prior to the onset of slipping, the shear stress acting on the interface exists only between the outer loading pins, whereas resistance to slipping acts over the entire length of the bar. Thus, slipping should initially occur simultaneously between the loading pins where the shear stress is constant and then spread outward as the load is increased and a shear force is developed outside of the loading pins. The additional load necessary for the slip zones to extend to the ends of the bar increases with the length-to-span ratio and the span-to-depth ratio. Thus, minimizing the length-to-span ratio should minimize the extent of the transition region and, hence, minimize the error in the calculation of energy absorption. Neglecting this transition region does not result in errors in determining the sliding resistance because the sliding resistance is determined from the first point of deviation from nonlinearity; the errors result only in predicting the hysteresis energy. In situations where the length-to-span ratio is large, it is possible to determine the energy absorption from the sliding resistance by calculating the load-deflection response, as described by Steif and Trojnacki. ${ }^{13}$

\section{(3) Significance of Energy Absorption during Frictional Sliding}

Previous studies have shown that layered materials can absorb a significant amount of energy during a flexural test. ${ }^{14,15}$ Folsom et al. ${ }^{6}$ and Phillipps et al..$^{16}$ have attributed the enhanced energy dissipation in these materials to the increased interfacial crack area that is created when crack deflection occurs at weak interfaces between the layers. ${ }^{16}$ The contribution from increased interfacial crack area to the total energy absorbed (the work-offracture or WOF) during a flexure test can be determined by multiplying the interfacial crack area by the interfacial fracture resistance. If the only mechanism for energy dissipation is the creation of interfacial crack area, the WOF and the calculated energy absorption should agree.

Phillipps et $a l .{ }^{14}$ have reported that the measured WOF during a flexural test for a typical silicon carbide-graphite layered specimen is $\sim 50 \mathrm{~mJ}$ for a specimen containing 15 layers. The maximum in the contribution to energy absorption by the creation of crack area would occur if every layer completely delaminated, which would lead to the absorption of $28 \mathrm{~mJ}$. However, observations suggest that delamination cracking is usually incomplete, and delamination does not occur at every interface; therefore, the actual contribution to energy absorption from interfacial cracking is even less. ${ }^{17,18}$ Similarly, Folsom et al. ${ }^{19}$ have shown that the WOF for a six-layer composite made from alumina sheets and fiber-reinforced epoxy is $\sim 0.60 \mathrm{~J}$. The measured interfacial fracture energy between the alumina and the fiber-reinforced epoxy is $155 \mathrm{~J} / \mathrm{m}^{2}$, yielding a maximum total contribution from interfacial cracking of $\sim 0.23 \mathrm{~J}$. The discrepancies between the measured WOF and the energy absorption calculated from the creation of interfacial crack area indicate that an additional source of energy dissipation must exist.

As we have shown, frictional sliding between cracked layers is a potent source of energy dissipation that may account for this discrepancy. The energy dissipated by a specimen containing multiple cracks can be calculated by multiplying the sliding resistance by the displacement of each layer. If it is assumed that the silicon carbide-graphite system that was tested by Phillipps et $a l .{ }^{14}$ had a sliding resistance similar to that of the silicon nitride-boron nitride system, an average sliding distance of $300 \mu \mathrm{m} /$ layer would be necessary to account for the observed 
energy dissipated during the flexural test. Experimental evidence suggests that sliding distances of this magnitude are commonly observed in layered materials. ${ }^{17,20}$

\section{Conclusions}

A simple method for measuring interfacial sliding resistance between cracked laminae in a layered ceramic has been developed. This method has been evaluated by measuring the frictional sliding resistance of model systems made from steel, and of a silicon nitride-boron nitride layered ceramic. Using this sliding resistance, it is possible to compute the energy dissipated during a load-unload cycle of a specimen containing a single crack. The amount of energy dissipated depends on the frictional sliding resistance, geometry of the specimen, and magnitude of the applied load. The predicted results are in good agreement with the observed hysteresis loops. Lastly, based on the measured hysteresis of the silicon nitride-boron nitride sample, it has been suggested that frictional sliding is a potent source of energy dissipation during flexural loading of layered materials. It may contribute to the substantial energy-absorption capabilities of these materials.

\section{Appendix}

To minimize friction between the loading roller pins and the specimen, a fully articulating testing apparatus with free-rolling pins has been used for the sliding experiments. Unfortunately, this articulation also introduces a significant amount of machine compliance into the loading system. In addition to storing elastic energy that must be dissipated by the specimen, the machine compliance also leads to errors in the calculation of sliding resistance and energy dissipation if it is not accounted for because the measured displacements at a given load are larger than the predicted displacements. By measuring the load-point specimen deflection rather than the crosshead deflection, a significant amount of machine compliance is accounted for, leaving only machine displacement located below the specimen in the load train.

Machine compliance located below the specimen has been determined by measuring the load-deflection response of a monolithic block of steel with a height of $5 \mathrm{~mm}$ and a width of $3.65 \mathrm{~mm}$. The difference between the measured compliance and theoretical compliance calculated from the known Young's modulus and specimen dimensions is attributed to machine compliance. The machine compliance is determined assuming that the specimen compliance and machine compliance act in series from

$$
m_{\mathrm{m}}=\left(\frac{1}{m_{\mathrm{s}}}-\frac{1}{m_{\mathrm{t}}}\right)^{-1}
$$

where $m_{\mathrm{m}}$ is the machine compliance, $m_{\mathrm{s}}$ the experimentally measured specimen compliance, and $m_{\mathrm{t}}$ the theoretical compliance of the specimen. When comparing theoretical predictions for the load-deflection response to measured load-deflection response, the machine stiffness (the inverse of the compliance) is simply added in the same manner shown in Eq. (9) to the theoretical stiffness in the sticking and sliding regimes $\left(m_{\mathrm{k}}\right.$ and $m_{\mathrm{p}}$, respectively).

Acknowledgments: The authors are grateful to John W. Halloran for his help in this work. The experimental assistance of Timothy Rickert in the preparation of the silicon nitride-boron nitride specimen and helpful discussions with Mohan Menon also are appreciated.

\section{References}

${ }^{1}$ D. B. Marshall, "An Indentation Method for Measuring Matrix-Fiber Frictional Stresses in Ceramic Composites," J. Am. Ceram. Soc., 67, C-259 (1984).

${ }^{2}$ D. B. Marshall and W. C. Oliver, "Measurement of Interfacial Mechanical Properties in Fiber-Reinforced Ceramic Composites," J. Am. Ceram. Soc., 70 [8] 542-48 (1987).

${ }^{3}$ D. R. Mumm and K. T. Faber, "Interfacial Debonding and Sliding in BrittleMatrix Composites Measured Using an Improved Fiber Pullout Technique," Acta Metall. Mater., 43 [3] 1259-70 (1995).

${ }^{4}$ E. Vagaggini, J.-M. Domergue, and A. G. Evans, "Relationships between Hysteresis Measurements and the Constituent Properties of Ceramic-Matrix Composites: I, Theory," J. Am. Ceram. Soc., 78 [10] 2709-20 (1995).

${ }^{5}$ A. G. Evans and F. W. Zok, "Review-The Physics and Mechanics of FibreReinforced Brittle-Matrix Composites," J. Mater. Sci., 29, 3857-96 (1994).

${ }^{6}$ C. A. Folsom, F. W. Zok, and F. F. Lange, "Flexural Properties of Brittle Multilayer Materials: I, Modeling," J. Am. Ceram. Soc., 77 [3] 689-96 (1994).

${ }^{7}$ E. P. Popov, Introduction to Mechanics of Solids; pp. 232-33. Prentice-Hall, Englewood Cliffs, NJ, 1968.

${ }^{8}$ M. D. Thouless, "Fracture of a Model Interface Under Mixed-Mode Loading," Acta Metall. Mater., 38 [6] 1135-40 (1990).

${ }^{9}$ D. Kovar, G. A. Brady, M. D. Thouless, and J. W. Halloran, "Interfacial Fracture Between Boron Nitride and Silicon Nitride and Its Applications to the Failure Behavior of Fibrous Monolithic Ceramics"; pp. 243-48 in Instability Dynamics, Scaling, and Ductile/Brittle Behavior, Vol. 409, Materials Research Society Fall Meeting, Symposium Q (Boston, MA, 1995). Edited by R. L. B. Selinger, J. J. Mecholsky, A. E. Carlsson, and E. R. Fuller Jr. Materials Research Society, Pittsburgh, PA.

${ }^{10}$ T. J. Mackin, P. D. Warren, and A. G. Evans, "Effects of Fiber Roughness on Interface Sliding in Composites," Acta Metall. Mater., 40, 1251-57 (1992).

${ }^{11}$ T. A. Parthasarathy, D. R. Barlage, P. D. Jero, and R. J. Kerans, "Effect of Interfacial Roughness Parameters on the Fiber Pushout Behavior of a Model Composite," J. Am. Ceram. Soc., 77 [12] 3232-36 (1994).

${ }^{12}$ S. Kumaria, R. N. Singh, and V. Gupta, "Effect of Interfacial Shear Strength on Crack-Fiber Interaction Behavior in Ceramic-Matrix Composites," J. Am. Ceram. Soc., 79 [1] 199-208 (1996).

${ }^{13}$ P. S. Steif and A. Trojnacki, "Bending Stress Enhancement in Materials with Limited Shear Resistance-Part I. Slipping-Layers Model," Int. J. Solids Struct., 30 [10] 1355-68 (1993).

${ }^{14}$ A. J. Phillipps, W. J. Clegg, and T. W. Clyne, "Fracture Behavior of Ceramic Laminates in Bending - II. Comparison of Model Predictions with Experimental Data," Acta Metall. Mater., 41 [3] 819-27 (1993).

${ }^{15}$ S. Baskaran and J. W. Halloran, "Fibrous Monolithic Ceramics: II, Flexural Strength and Fracture Behavior of the Silicon Carbide/Graphite System," J. Am. Ceram. Soc., 76 [9] 2217-24 (1993).

${ }^{16}$ A. J. Phillipps, W. J. Clegg, and T. W. Clyne, "Fracture Behaviour of Ceramic Laminates in Bending-I. Modeling of Crack Propagation," Acta Metall. Mater., 41 [3] 805-17 (1993).

${ }^{17}$ W. J. Clegg, K. Kendall, N. McN. Alford, T. W. Button, and J. D. Birchall, "A Simple Way to Make Tough Ceramics," Nature (London), 357 [Oct. 4] 45557 (1990).

${ }^{18}$ A. J. Phillipps, W. J. Clegg, and T. W. Clyne, "The Correlation of Interfacial and Macroscopic Toughness in SiC Laminates," Composites, 24 [2] 166-76 (1993).

${ }^{19} \mathrm{C}$. A. Folsom, F. W. Zok, and F. F. Lange, "Mechanical Behavior of a Laminar Ceramic-Fiber-Reinforced Epoxy Composite," J. Am. Ceram. Soc., 75 [11] 2969-75 (1992).

${ }^{20}$ C. A. Folsom, F. W. Zok, and F. F. Lange, "Flexural Properties of Brittle Multilayer Materials: II, Experiments," J. Am. Ceram. Soc., 77 [8] 2081-87 (1994). 